PROCEEDINGS OF THE AMERICAN MATHEMATICAL SOCIETY

Volume 124, Number 10, October 1996

\title{
A COUNTEREXAMPLE CONCERNING SMOOTH APPROXIMATION
}

\author{
CHRISTOPHER J. BISHOP
}

(Communicated by Theodore W. Gamelin)

\begin{abstract}
We answer a question of Smith, Stanoyevitch and Stegenga in the negative by constructing a simply connected planar domain $\Omega$ with no twosided boundary points and for which every point on $\Omega^{c}$ is an $m_{2}$-limit point of $\Omega^{c}$ and such that $C^{\infty}(\bar{\Omega})$ is not dense in the Sobolev space $W^{k, p}(\Omega)$.
\end{abstract}

\section{INTRODUCTION}

Suppose $\Omega \subset \mathbb{R}^{2}$ is simply connected. Let $C^{\infty}(\bar{\Omega})$ denote the restriction to $\Omega$ of $C^{\infty}\left(\mathbb{R}^{2}\right)$ and let $W^{k, p}(\Omega)$ denote the Sobolev space of functions on $\Omega$ defined by

$$
\|f\|_{W^{k, p}(\Omega)}=\|f\|_{L^{p}(\Omega)}+\sum_{|\alpha| \leq k}\left\|D^{\alpha} f\right\|_{L^{p}(\Omega)}<\infty .
$$

Let $\Omega^{c}=\mathbb{R}^{2} \backslash \Omega$ denote the complement of $\Omega$ and let $m_{1}, m_{2}$ denote linear and two-dimensional Lebesgue measures. We say that a point $z \in E$ is an $m_{2}$ limit point of $E$ if $m_{2}(E \cap B(z, r))>0$ for every disk centered at $z$. Also, $x \in \partial \Omega$ is called a two-sided boundary point of $\Omega$ if there is a $\delta(x)>0$ so that for every $0<\delta<\delta(x), B(x, \delta) \cap \Omega$ has at least two components whose closures contain $x$.

Meyers and Serrin [1] proved that $C^{\infty}(\Omega)$ is always dense in $W^{k, p}(\Omega)$, but it is known that $C^{\infty}(\bar{\Omega})$ need not be. In their paper [2] Smith, Stanoyevitch and Stegenga prove several interesting theorems describing when $C^{\infty}(\bar{\Omega})$ is dense in $W^{k, p}(\Omega)$ and give several examples where it is not dense. Based on their results they asked the following.

Question. If $\Omega$ is a simply connected planar domain without two-sided boundary points and for which all points in $\Omega^{c}$ are $m_{2}$-limit points of $\Omega^{c}$, then does it follow that $C^{\infty}(\bar{\Omega})$ is dense in $W^{k, p}(\Omega)$ ?

The purpose of this note is to show the answer is no.

\section{The CONSTRUCTION}

We start by constructing a family of Cantor sets $E(t) \subset[0,1]$. These sets will depend continuously on $t \in[0,1]$ and will satisfy $m_{1}(E(t))=t$. To begin, let

Received by the editors November 23, 1994 and, in revised form, April 3, 1995.

1991 Mathematics Subject Classification. Primary 46E35.

Key words and phrases. Sobolev spaces, smooth approximation.

The author is partially supported by NSF Grant DMS 92-04092 and an Alfred P. Sloan research fellowship.

(C)1996 American Mathematical Society 
$E_{0}(t)=[0,1]$ and let $I_{0}(t)$ be the open interval of length $\frac{1}{2}(1-t)$ centered at $\frac{1}{2}$. Then $E_{1}(t)=[0,1] \backslash I_{0}(t)$ consists of 2 closed intervals each of length $\frac{1}{2}\left(1-\frac{1}{2}(1-t)\right)=$ $\frac{1}{4}(1+t)$. Let $I_{2}(t)$ be the union of the two open intervals, each of length $\frac{1}{8}(1-t)$ and concentric with the components of $E_{1}(t)$. Let $E_{2}(t)=E_{1}(t) \backslash I_{2}(t)$. Continue in this way, obtaining a sequence of nested compact sets $E_{1}(t) \supset E_{2}(t) \supset \cdots \supset E_{n}(t) \supset$ ... where $E_{n}(t)=E_{n-1}(t) \backslash I_{n}(t)$ consists of $2^{n}$ closed intervals, each of length $2^{-n}\left(1-\left(1-2^{-n}\right)(1-t)\right)$. The intersection of these sets is a Cantor set $E(t)$ of linear measure $t$.

Let $F$ be any Cantor set of linear measure 1 in $[-1,1]$, say $F=2 E(1 / 2)-1$, so that $m_{1}(F)=1$. Let

$$
K=\left\{(x, y):-2 \leq x \leq 2, y \in E\left(\min \left(\frac{1}{2}, \operatorname{dist}(x, F)\right)\right)\right\} .
$$

See Figure 2.1. It may help to visualize the set if we note that $K$ is homeomorphic to a Cantor set times an interval. The vertical cross sections of $K$ are all sets of the form $E(t)$. For $x \in\left[-2,-\frac{3}{2}\right] \cup\left[\frac{3}{2}, 2\right]$ the cross section is $E(1 / 2)$ and for $x \in F$ it is $E(0)$. Since $K$ has vertical cross section of positive linear measure for a open dense set of $x$ 's, $K$ has positive area and every point of $K$ is an $m_{2}$-limit of $K$.
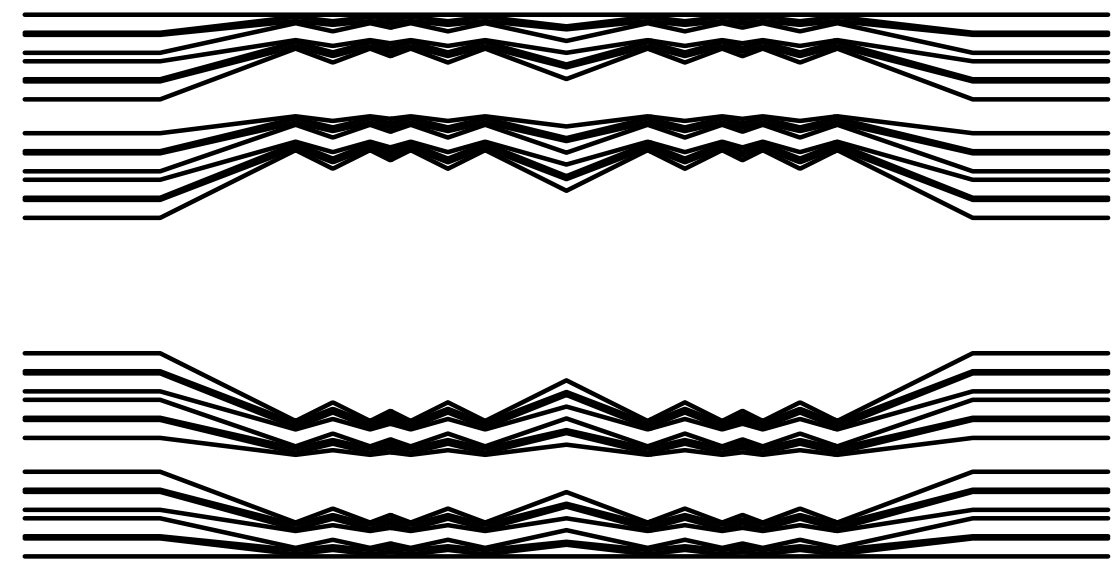

Figure 2.1. The set $K$.

The set $K$ is not connected, and we add rectangles to make it so. More precisely, for $n$ even let

and for $n$ odd define

$$
R_{n}=\left[-2,-\frac{3}{2}\right] \times I_{n}(1 / 2),
$$

$$
R_{n}=\left[\frac{3}{2}, 2\right] \times I_{n}(1 / 2) .
$$

Set $J=K \cup \bigcup_{n} R_{n}$. Each "horizontal tube" in the complement of $K$ is now blocked by exactly one rectangle, so $J$ is connected.

Let $\Omega=(-3,3) \times(0,3) \backslash J$. See Figure 2.2. It is easy to check that $\Omega$ is simply connected, has no two-sided boundary points and every point of $\Omega^{c}$ is an $m_{2}$-limit of $\Omega^{c}$. Thus it only remains to show that $C^{\infty}(\bar{\Omega})$ is not dense in $W^{k, p}(\Omega)$. In fact, by Hölder's inequality we need only show it is not dense in $W^{1,1}(\Omega)$.

Let $\Omega_{0} \subset \Omega$ be the component of $\Omega \cap\{(x, y):-1<x<1\}$ which contains the point $(0,2)$. On $\Omega_{0}$ let $f(x, y)=x$. On the two components $\Omega_{+}, \Omega_{-}$of $\Omega \backslash \Omega_{0}$ let 
$f$ be the constant \pm 1 , chosen to make $f$ continuous. We claim that this function cannot be approximated in $W^{1,1}(\Omega)$ by elements of $C^{\infty}(\bar{\Omega})$.

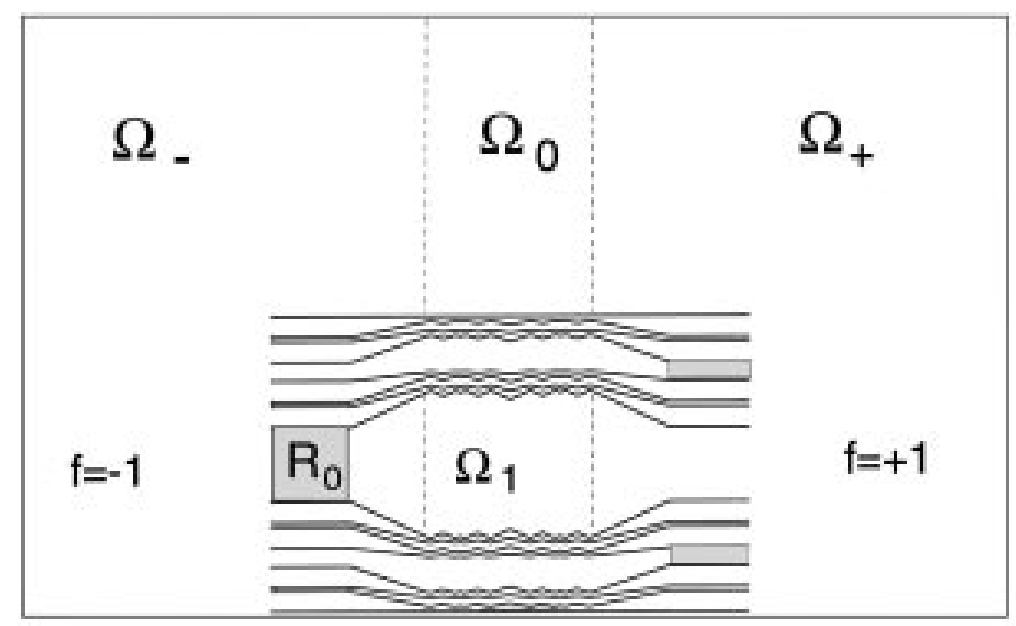

Figure 2.2. $\Omega$ and $f$.

Suppose $g \in C^{\infty}(\bar{\Omega})$. Let $\Omega_{1}$ be the component of $\Omega \cap\{(x, y):-1 \leq x \leq 1\}$ which contains the point $\left(0, \frac{1}{2}\right)$. By the definition of $E(t)$, we see that $\Omega_{1} \subset \Omega_{+}$ and

$$
\left\{(x, y):-1<x<1, \frac{3}{8}<y<\frac{5}{8}\right\} \subset \Omega_{1} \subset\left\{(x, y):-1<x<1, \frac{1}{4} \leq y \leq \frac{3}{4}\right\} .
$$

Thus $f$ is the constant 1 on $\Omega_{1}$, so for any $\delta>0$ there is an $\epsilon>0$ such that

$$
\int_{\Omega}|f-g| d x d y<\epsilon
$$

implies $g>1 / 2$ on a set $S \subset \Omega_{1}$ of measure $\geq(1-\delta) m_{2}\left(\Omega_{1}\right)$. If $\delta$ is small enough then the vertical projection of $S$ must hit $F$ in a set $F^{\prime}$ of measure at least $\frac{1}{2} m_{1}(F)=\frac{1}{2}$.

Write $F^{\prime}=F_{1} \cup F_{2}$ where

$$
\begin{gathered}
F_{1}=\left\{x \in F^{\prime}: g(x, y) \geq 0 \text { for all } 0 \leq y \leq \frac{3}{4}\right\}, \\
F_{2}=\left\{x \in F^{\prime}: g(x, y) \leq 0 \text { for some } 0 \leq y \leq \frac{3}{4}\right\} .
\end{gathered}
$$

One of these two sets must have measure greater than $\frac{1}{4} m_{1}(F)=\frac{1}{4}$.

First suppose $m\left(F_{1}\right) \geq \frac{1}{4}$. Then $|f-g| \geq 1$ on the set $\left(F_{1} \times[0,1 / 4]\right) \cap \Omega_{-}$. Thus

$$
\|f-g\|_{W^{1,1}(\Omega)} \geq \int_{\Omega}|f-g| d x d y \geq m_{2}\left(\left(F_{1} \times[0,1 / 4]\right) \cap \Omega_{-}\right)>0,
$$

independent of $g$.

On the other hand, suppose $m_{1}\left(F_{2}\right) \geq 1 / 4$. If $x \in F_{2}$, then $g$ varies by at least $1 / 2$ on the segment $I_{x}=\{x\} \times[0,3 / 4]$ so

$$
\int_{0}^{3 / 4}\left|\frac{\partial g}{\partial y}(x, y)\right| d y \geq 1 / 2
$$


Since $\nabla f=0$ on $\Omega \backslash \Omega_{0}$, we get

$$
\|f-g\|_{W^{1,1}(\Omega)} \geq \int_{\left(F_{2} \times[0,3 / 4]\right) \cap \Omega}\left|\frac{\partial g}{\partial y}(x, y)\right| d x d y .
$$

Since $F_{2} \subset F, x \in F_{2}$ implies $m_{1}\left(\Omega \cap I_{x}\right)=m_{1}\left(I_{x}\right)$; this is where we use the fact the vertical cross sections of $K$ have zero length when $x \in F$. Hence

and so

$$
\int_{\left(F_{2} \times[0,3 / 4]\right) \cap \Omega}\left|\frac{\partial g}{\partial y}(x, y)\right| d y=\int_{F_{2} \times[0,3 / 4]}\left|\frac{\partial g}{\partial y}(x, y)\right| d y
$$

$$
\|f-g\|_{W^{1,1}(\Omega)} \geq \int_{F_{2} \times[0,3 / 4]}\left|\frac{\partial g}{\partial y}(x, y)\right| d y \geq \frac{1}{2} m_{1}\left(F_{2}\right) \geq \frac{1}{8} .
$$

Therefore in both cases we have shown that $\|f-g\|_{W^{1,1}(\Omega)}$ is bounded away from zero with an estimate independent of $g$. This proves that $C^{\infty}(\bar{\Omega})$ is not dense in $W^{1,1}(\Omega)$ and hence not dense in any $W^{k, p}(\Omega)$ for $k \geq 1$ or $1 \leq p<\infty$.

\section{ACKNOWLEDGEMENTS}

I thank the referee for several helpful remarks.

\section{REFERENCES}

1. N. Meyers and J. Serrin, $H=W$, Proc. Nat. Acad. Sci. USA 51 (1964), 1055-1056. MR 29:1551

2. W. Smith, A. Stanoyevitch, and D.A. Stegenga, Smooth approximation of Sobolev functions on planar domains, J. London Math. Soc. 49 (1994), 309-330. MR 95e:46043

Department of Mathematics, SUny at Stony Brook, Stony Brook, New York 117943651

E-mail address: bishop@math.sunysb.edu 